\title{
Imbalanced mobilities causing S-shaped IV curves in planar heterojunction organic solar cells
}

\author{
Wolfgang Tress, ${ }^{\text {a) }}$ Annette Petrich, Markus Hummert, Moritz Hein, Karl Leo, and \\ Moritz Riede \\ Institut für Angewandte Photophysik, Technische Universität Dresden, George-Bähr-Str. 1, \\ 01069 Dresden, Germany
}

(Received 23 December 2010; accepted 13 January 2011; published online 8 February 2011)

\begin{abstract}
We show that S-kinks in the current voltage characteristics, which decrease the fill factor significantly, can be caused by a strong imbalance of charge carrier mobilities (hole mobility in donor and electron mobility in acceptor) in planar/flat heterojunction organic solar cells. Electrical simulations according to a drift-diffusion model predict the occurrence of an S-kink for a mobility mismatch factor larger than 100 . By combining a low-mobility donor material, $\left(1,2,3,4,9,10,11,12\right.$-octaphenyl-diindeno[1,2,3-cd:1', $2^{\prime}, 3^{\prime}$-lm]perylene), with the acceptors $\mathrm{C}_{60}$ and $\mathrm{N}, \mathrm{N}^{\prime}$-dimethylperylene-3,4:9,10-dicarboximide, which show different electron mobilities, we experimentally verify the predictions. Our results demonstrate that not only interface effects but also the photoactive material itself can cause S-kinks. (C) 2011 American Institute of Physics.
\end{abstract}

[doi:10.1063/1.3553764]

The development of organic solar cells quickly progressed in the last decade. Besides many theoretical studies, the synthesis of tailored molecules has mainly helped to increase the power conversion efficiency significantly. However, when investigating new materials, the current-voltage characteristics (IV curves) frequently show undesirable S-kinks, which reduce the fill factor $(F F)$ and hence the efficiency of a material system considerably. The reasons are not well understood, and several explanations have been given. A key question is whether the S-kink is caused by the active material itself or by its interface to the electrode. Commonly, S-kinks are attributed to barriers at the contacts. ${ }^{1-3}$ Some simulation data in the literature indicate that low charge carrier mobilities could also cause S-kinks. ${ }^{4}$ However, systematic investigations of this issue are lacking, and the effect has not been unambiguously proven experimentally. Furthermore, the effect presented here has to be distinguished from space charge limited photocurrents in polymer bulk heterojunctions (BHJs), caused by imbalanced mobilities. ${ }^{5}$

Here, we show that imbalanced mobilities can cause S-shapes by comparing drift-diffusion simulations of bilayer devices with systematic mobility variations to experimental data of flat heterojunction (FHJ) solar cells. Simulated IV curves are obtained solving transport, continuity, and Poisson's equations self-consistently with an algorithm ${ }^{3}$ based on Staudigel et al. ${ }^{6}$ This approach allows for considering space charge effects that will play an important role in the following discussion. The donor/acceptor (D/A) interface is implemented as source of free charge carrier generation, which is for simplicity assumed to happen instantaneously by exciton separation and dissociation after exciton diffusion to the interface. We do not consider the controversially discussed ${ }^{7}$ path via charge transfer states, because it does not affect the conclusions of this letter. Besides being the source of charge carrier generation, the interface is the only significant locus of free charge carrier recombination, as photogenerated and

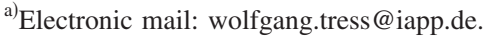

injected holes are mainly located on the donor and electrons on the acceptor. We assume bimolecular recombination and find that changing the recombination mechanism to, e.g., Shockley-Read-Hall or geminate recombination does not change the S-kink in the simulated IV curves qualitatively. Hence, knowing the exact recombination mechanism is also not essential here.

Devices are fabricated by consecutive evaporation of purified small molecule organic materials in vacuum. The active layers are sandwiched between doped charge transport layers providing Ohmic contact to the electrodes. ${ }^{8}$ The stack consists of indium tin oxide (ITO)/p-doped (9,9-bis4-[di-(p-biphenyl)aminophenyl]fluorene) (BPAPF) $(25 \mathrm{~nm}, 10$ wt \%)/(1,2,3,4,9,10,11,12-octaphenyl-diindeno [1,2,3-cd: 1' $, 2^{\prime}, 3^{\prime}$-lm]perylene) (Ph4-Ph4-DIP) (10, 20, $40,60 \mathrm{~nm}) /\left[\mathrm{C}_{60}\right.$ or Me-PTCDI $\left.(30 \mathrm{~nm})\right] / \mathrm{n}-\mathrm{C}_{60}(20 \mathrm{~nm}$, 3 wt \%)/Al. BPAPF is the hole transport material, $\mathrm{Ph} 4-\mathrm{Ph} 4-$ DIP is the donor, and $\mathrm{C}_{60}$ and $\mathrm{N}, \mathrm{N}^{\prime}$-dimethylperylene3,4:9,10-dicarboximide (Me-PTCDI) are applied as acceptors. As dopants NDP9 and NDN1 (Novaled AG, Dresden, Germany) are used. IV curves are measured under illumination of simulated sunlight (SC1200, KHS Technical Lighting, Germany) with an intensity of $100, \ldots, 110 \mathrm{~mW} / \mathrm{cm}^{2}$ monitored by a calibrated silicon photodiode and corrected for spectral mismatch.

Before discussing the experimental data, simulation data for an exemplary model device are analyzed. It consists of a bilayer with thermionic contacts with insignificant injection barriers $(\leq 0.1 \mathrm{eV})$. Donor and acceptor thicknesses are both chosen as $30 \mathrm{~nm}$, which is a common value for small molecule organic solar cells. Figure 1 shows the calculated IV curves for a variation of hole mobility in the donor $\left(\mu_{h}^{D}\right)$ and electron mobility in the acceptor $\left(\mu_{e}^{A}\right)$. For better comparability of the curves, the recombination constant for bimolecular recombination is set independent of mobility to a value of $5 \times 10^{-10} \mathrm{~cm}^{3} / \mathrm{s}$, which is expected when using Langevin theory at a mobility of $10^{-3} \mathrm{~cm}^{2} / \mathrm{V} \mathrm{s}$. Using the Langevin expression has a minor effect on the qualitative shape of the IV curve. However, the open circuit voltage $\left(V_{\mathrm{OC}}\right)$ signifi- 


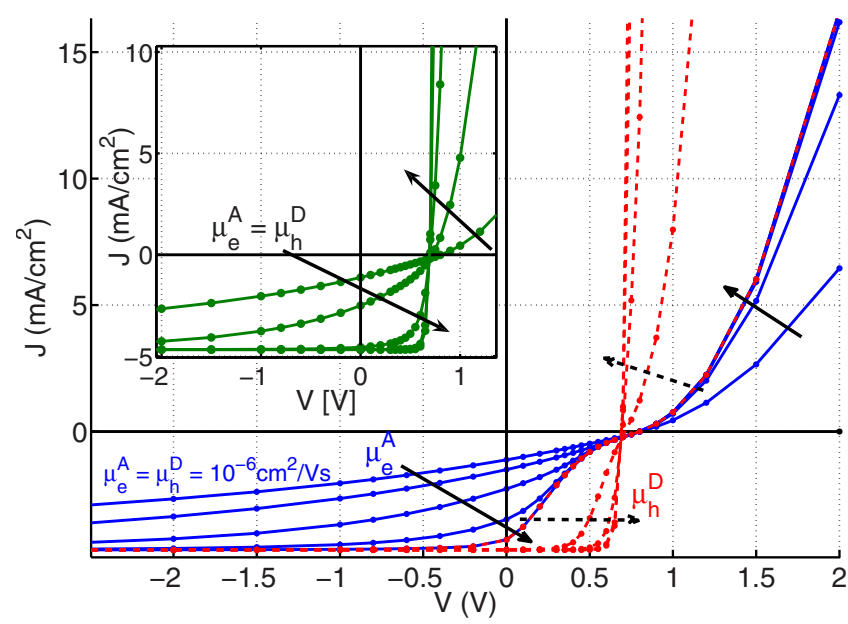

FIG. 1. (Color online) Simulation data of a bilayer device with varied mobilities. Solid lines: hole mobility in donor $\mu_{h}^{D}$ kept constant at $10^{-6} \mathrm{~cm}^{2} / \mathrm{V} \mathrm{s}$, with electron mobility in acceptor $\mu_{e}^{A}$ varied $\left(10^{-6}, 10^{-5}, 10^{-4}, 10^{-3}, 10^{-2} \mathrm{~cm}^{2} / \mathrm{V} \mathrm{s}\right)$. Dashed lines: $\mu_{e}^{A}$ constant at $10^{-2} \mathrm{~cm}^{2} / \mathrm{V} \mathrm{s}$, with $\mu_{h}^{D}$ varied $10^{-6}, \ldots, 10^{-2} \mathrm{~cm}^{2} / \mathrm{V} \mathrm{s}$. Inset: balanced mobilities $\mu_{e}^{A}=\mu_{h}^{D}=10^{-6}, \ldots, 10^{-2} \mathrm{~cm}^{2} / \mathrm{V}$ s. Details can be found in the supplementary material (Ref. 9).

cantly changes with recombination probability, which makes a comparable visualization of the strength of the S-kink difficult. The simulations show that high mobilities lead to high $F F$, whereas low mobilities decrease the $F F$ strongly. An S-kink appears for imbalanced mobilities at a mobility mismatch $\mu_{e}^{A} / \mu_{h}^{D}$ (or $\mu_{h}^{D} / \mu_{e}^{A}$, respectively) of $\approx 100, \ldots, 1000$. The S-kink cannot be seen when both mobilities are balanced and low (inset). Simulations including varied layer thicknesses show that the strength of the S-kink scales with the thickness ratio of low-mobility to high-mobility layer and is less influenced by the total device thickness. ${ }^{9}$ The S-kink gets more pronounced for a thicker low-mobility layer compared to the thickness of the high-mobility layer. Regarding device performance, imbalanced high/low mobilities are preferable compared to balanced low mobilities, which show lower power conversion efficiencies than the S-kink curves in Fig. 1.

The S-kink in the calculations is only caused by transport properties of free charge carriers. Field-dependent exciton dissociation according to the Onsager-Braun theory ${ }^{10}$ does not change the shape significantly. The reason for the S-kink can be explained by examining profiles of charge carrier densities and the electric field distribution within the layers. Figure 2 shows data of three mobility pairs at two different applied voltages, 0 and $0.55 \mathrm{~V}$, which represents the S-kink region. For balanced high mobilities, the electric field (at zero bias originating from the built-in voltage) in the device is constant. For both voltages, all charge carriers are extracted due to their high mobilities. The driving force is mainly drift due to the built-in field assisted by diffusion away from the interface. For very low mobilities, a space charge is built up in the complete device, because electrons and holes cannot be extracted sufficiently fast. Their concentration at the D/A interface is increased and hence recombination probability as well, which leads to a lower $F F$. In the imbalanced mobility case, the field distribution is asymmetric, because only the less mobile holes form a space charge. This reduces the drop of the electrical potential in the acceptor and increases the magnitude of the field in the donor,
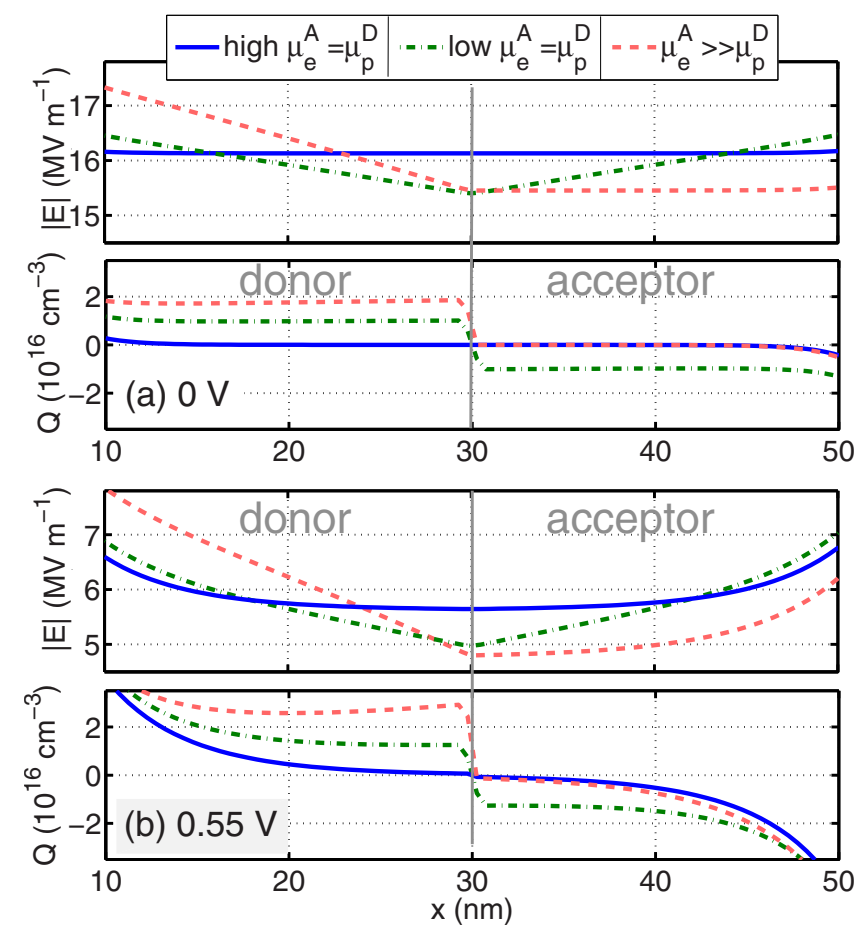

FIG. 2. (Color online) Electric field $E$ and charge density $Q$ per elementary charge as functions of distance $x$ from anode. (a) at $0 \mathrm{~V}$ and (b) at $0.55 \mathrm{~V}$ applied bias in the model device of Fig. 1 for high mobilities $\mu_{e}^{A}=\mu_{h}^{D}$ $=10^{-2} \mathrm{~cm}^{2} / \mathrm{V} \mathrm{s}$ (solid lines), for low mobilities $\mu_{e}^{A}=\mu_{h}^{D}=10^{-5} \mathrm{~cm}_{e}^{2} / \mathrm{V} \mathrm{s}$ (dash-dotted lines), and for highly imbalanced mobilities $\mu_{e}^{A}$ $=10^{-2} \mathrm{~cm}^{2} / \mathrm{V} \mathrm{s}, \mu_{h}^{D}=10^{-5} \mathrm{~cm}^{2} / \mathrm{V} \mathrm{s}$ (dashed lines).

which assures that for every extracted (fast) electron, a hole is extracted in steady state. This rearrangement of the field guarantees the extraction of every photogenerated charge carrier at $0 \mathrm{~V}$. However, with decreasing field, resulting from positive applied bias [Fig. 2(b)], electrons start to pile up at the D/A interface as well. Recombination is increased and hence current is decreased, so that the IV curve approaches the low-mobility curve, resulting in an S-kink. Such an S-kink is not expected in a bulk heterojunction, because the field-free region (resulting from the $\mu$-imbalance) close to the contact of the more mobile charge carrier, where no photocurrent is collected, decreases continuously with negative applied bias. This leads to a smooth increase of photocurrent.

The following items have to be checked for an experimental verification of the simulation results: (i) The IV curve of a FHJ comprising a D/A combination with a high mobility mismatch and with Ohmic contacts shows an S-kink. (ii) The thickness dependence of the S-kink matches the predictions of simulation. (iii) The S-kink disappears with a decreased imbalance in mobility. (iv) The S-kink vanishes in a blend without changing $V_{\mathrm{OC}}$.

The donor material Ph4-Ph4-DIP (Ref. 11) [Fig. 3(a)] is chosen to prove these points. It is synthesized as substitute for the commonly used $\mathrm{ZnPc}$ with the aim of shifting the absorption from the red into the green spectral range accompanied by a higher $V_{\mathrm{OC}}$. Both goals are realized. ${ }^{9}$ However, the contribution to the photocurrent in a FHJ with $\mathrm{C}_{60}$ is low due to a low exciton diffusion length. ${ }^{9}$ As mobility and exciton diffusion length are expected to follow the same trend (for Dexter transfer), a low $\mu_{h}$ can be expected. Single carrier device data with layer thicknesses between 10 and $60 \mathrm{~nm}$ suggest a strong field $(E)$-dependent 


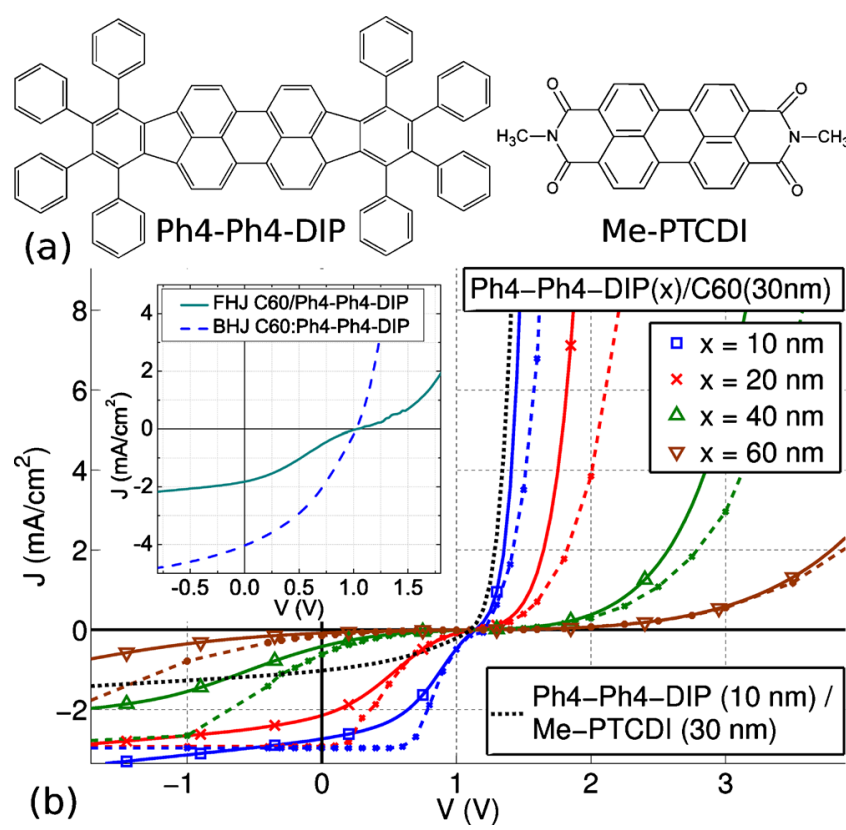

FIG. 3. (Color online) (a) Molecular structure of $\mathrm{Ph} 4-\mathrm{Ph} 4-\mathrm{DIP}$ and Me-PTCDI. (b) Solid lines: experimental data of devices ITO/p-BPAPF/Ph4-Ph4-DIP/(C 60 or Me-PTCDI $) / \mathrm{n}^{-\mathrm{C}_{60}} / \mathrm{Al}$. $F F$ is $41 \%$ for $10 \mathrm{~nm} \mathrm{Ph} 4-\mathrm{Ph} 4-\mathrm{DIP} / \mathrm{C}_{60}$ and $37 \%$ for $10 \mathrm{~nm} \mathrm{Ph} 4-\mathrm{Ph} 4-\mathrm{DIP} / \mathrm{Me}-\mathrm{PTCDI}$. Dashed lines: simulation data. Inset shows FHJ and BHJ in inverted architecture: ITO/C 60 (20 nm)/active layer/Ph4-Ph4-DIP (5 nm)/p-BPAPF (40 $\mathrm{nm}) / \mathrm{p}-\mathrm{ZnPc}(10 \mathrm{~nm}) / \mathrm{Au}(40 \mathrm{~nm})$ with active layer $\mathrm{C}_{60}(5 \mathrm{~nm}) / \mathrm{Ph} 4-\mathrm{Ph} 4-\mathrm{DIP}$ $(25 \mathrm{~nm})$ for $\mathrm{FHJ}$ and $\mathrm{C}_{60}$ : Ph4-Ph4-DIP (25 nm; 3:2) for BHJ.

mobility in the range of $\mu_{h}=0.5, \ldots, 5 \exp [5.5, \ldots, 6$ $\left.\times 10^{-3}(\mathrm{~cm} / \mathrm{V})^{1 / 2} E^{1 / 2}\right] 10^{-8} \mathrm{~cm}^{2} / \mathrm{V} \mathrm{s}$. The reason for such a low value could be the phenyl end groups of the molecule, which are free to rotate out of plane, hindering close stacking and hence $\pi$-electron wave function overlap between molecules. The mobility of $\mathrm{C}_{60}$ in a thin film is reported to be in the range of $10^{-2} \mathrm{~cm}^{2} / \mathrm{V} \mathrm{s} .{ }^{12}$ Comparing these mobility data to the predictions of simulation (Fig. 1), an S-kink is expected for a FHJ $\mathrm{Ph} 4-\mathrm{Ph} 4-\mathrm{DIP} / \mathrm{C}_{60}$ and is indeed observed [Fig. 3(b)]. A simulation (dashed lines) of the complete solar cell stack with the mentioned mobilities and without any parameter optimization describes the shape of the S-kink around $V_{\mathrm{OC}}$ well, including the strong dependence on donor thickness. This fact and the good alignment of the hole transport levels of BPAPF (Ref. 3) and Ph4-Ph4-DIP (both show a $V_{\mathrm{OC}}$ of $1, \ldots, 1.1 \mathrm{~V}$ in a $\mathrm{FHJ}$ with acceptor $\mathrm{C}_{60}$; further measurements are in Ref. 9) exclude barriers at the contacts as reason for the S-kink. Increasing $\mu_{h}^{D}$ by replacing $\mathrm{Ph} 4-$ $\mathrm{Ph} 4-\mathrm{DIP}$ with $\mathrm{ZnPc}$ leads to well known $\mathrm{ZnPc} / \mathrm{C}_{60}$ solar cells without $\mathrm{S}$-shapes. ${ }^{3}$ It is more interesting to investigate a decrease of $\mu_{e}^{A}$ by using Me-PTCDI, a material with a comparable electron affinity to $\mathrm{C}_{60}$. However, organic field effect transistor (OFET) measurements (in bottom-gate geometry with $\mathrm{SiO}_{2}$-dielectrics and Au contacts) show an electron mobility, which is significantly lower than in $\mathrm{C}_{60}\left(\mu_{e}\left(\mathrm{C}_{60}\right) \approx 7\right.$ $\times 10^{-3} \mathrm{~cm}^{2} / \mathrm{V} \mathrm{s} ; \quad \mu_{e} \quad($ Me-PTCDI $) \approx 2 \times 10^{-4} \mathrm{~cm}^{2} / \mathrm{V} \mathrm{s}$;
$\left.\mu_{h}(\mathrm{Ph} 4-\mathrm{Ph} 4-\mathrm{DIP}) \approx 2 \times 10^{-6} \mathrm{~cm}^{2} / \mathrm{V} \mathrm{s}\right)$. Although the OFET mobility is determined under different measurement conditions, rough trends can be transferred to layers in solar cell geometry. The dotted IV curve in Fig. 3 representing a $\mathrm{Ph} 4-$ $\mathrm{Ph} 4-\mathrm{DIP}(10 \mathrm{~nm}) / \mathrm{Me}-\mathrm{PTCDI}$ device shows no S-kink as predicted by simulation. However, $F F$ is lower than in the solar cell with $\mathrm{C}_{60}$ caused by the lower mobility of Me-PTCDI. The photocurrent is decreased mainly due to a smaller contribution from Me-PTCDI compared to $\mathrm{C}_{60}$. The lower photocurrent cannot explain the difference in the shape between $\mathrm{C}_{60}$ and Me-PTCDI as acceptor, because a varied illumination intensity does not influence the S-kink significantly. MePTCDI solar cells also show an S-kink for thicker Ph4-Ph4DIP layers, which fits to the predicted thickness dependence (data not plotted). Additionally, inverted cells are made (inset of Fig. 3), where the S-kink is also present. In the bulk heterojunction, however, there is no S-kink present at the same $V_{\mathrm{OC}}$. This excludes barriers at the contacts, which have been extensively discussed previously, ${ }^{3}$ as reason for the S-shape.

In conclusion, we have performed drift-diffusion simulations of bilayer devices showing S-kinks in the IV curve for an imbalance in charge carrier mobilities $\left(\mu_{e}^{A} / \mu_{h}^{D}\right)$ larger than 100 for comparable layer thicknesses. In this case, instead of the contacts, the charge transport properties of the active material itself are responsible for the S-kink. We verify this effect experimentally in flat heterojunction solar cells with a low-mobility donor combined with different acceptors.

W.T. thanks the Reiner Lemoine Foundation for funding. Additional support from BMBF (OPEG Grant No. 13N9720 and Innoprofile Grant No. 03IP602) is gratefully acknowledged.

${ }^{1}$ K. Schulze, C. Uhrich, R. Schueppel, K. Leo, M. Pfeiffer, E. Brier, E. Reinold, and P. Baeuerle, Adv. Mater. 18, 2872 (2006).

${ }^{2}$ H. Jin, M. Tuomikoski, J. Hiltunen, P. Kopola, A. Maaninen, and F. Pino, J. Phys. Chem. C 113, 16807 (2009).

${ }^{3}$ W. Tress, K. Leo, and M. Riede, "Influence of hole transport layers and donor materials on open circuit voltage and shape of IV curves of organic solar cells," Adv. Funct. Mater. (submitted).

${ }^{4}$ J. Nelson, J. Kirkpatrick, and P. Ravirajan, Phys. Rev. B 69, 035337 (2004).

${ }^{5}$ V. D. Mihailetchi, J. Wildeman, and P. W. M. Blom, Phys. Rev. Lett. 94, 126602 (2005).

${ }^{6}$ J. Staudigel, F. Steuber, M. Stoessel, and J. Simmerer, J. Appl. Phys. 86, 3895 (1999)

${ }^{7}$ J. Lee, K. Vandewal, S. R. Yost, M. E. Bahlke, L. Goris, M. A. Baldo, J. V. Manca, and T. V. Voorhis, J. Am. Chem. Soc. 132, 11878 (2010).

${ }^{8}$ M. Riede, T. Mueller, W. Tress, R. Schueppel, and K. Leo, Nanotechnology 19, 424001 (2008).

${ }^{9}$ See supplementary material at http://dx.doi.org/10.1063/1.3553764 for further information redarding modeling and additional characterization of $\mathrm{Ph} 4-\mathrm{Ph} 4-\mathrm{DIP}$ devices.

${ }^{10}$ V. Mihailetchi, L. Koster, J. Hummelen, and P. Blom, Phys. Rev. Lett. 93, 216601 (2004).

${ }^{11}$ M. Wehmeier, M. Wagner, and K. Muellen, Chem.-Eur. J. 7, 2197 (2001).

${ }^{12}$ B. P. Rand, J. G. Xue, S. Uchida, and S. R. Forrest, J. Appl. Phys. 98, $124902(2005)$. 